\title{
Continuing Bonds after Loss by Suicide: A Systematic Review
}

\author{
Rebecca Goodall ${ }^{1}$, Karolina Krysinska ${ }^{2}$ (1) and Karl Andriessen ${ }^{2, *} \mathbb{B}$ \\ 1 Melbourne Medical School, The University of Melbourne, Parkville, VIC 3010, Australia; \\ rngoodall@student.unimelb.edu.au \\ 2 Centre for Mental Health, Melbourne School of Population and Global Health, The University of Melbourne, \\ Parkville, VIC 3010, Australia; karolina.krysinska@unimelb.edu.au \\ * Correspondence: karl.andriessen@unimelb.edu.au
}

Citation: Goodall, R.; Krysinska, K.; Andriessen, K. Continuing Bonds

after Loss by Suicide: A Systematic

Review. Int. J. Environ. Res. Public

Health 2022, 19, 2963. https://

doi.org/10.3390/ijerph19052963

Academic Editor: Paul B. Tchounwou

Received: 29 January 2022

Accepted: 1 March 2022

Published: 3 March 2022

Publisher's Note: MDPI stays neutral with regard to jurisdictional claims in published maps and institutional affiliations.

Copyright: (c) 2022 by the authors. Licensee MDPI, Basel, Switzerland. This article is an open access article distributed under the terms and conditions of the Creative Commons Attribution (CC BY) license (https:/ / creativecommons.org/licenses/by/ $4.0 /)$.

\begin{abstract}
The concept of continuing bonds as an alternative to detachment from the deceased person has gained traction in grief literature over the years. Those bereaved by suicide are likely to experience various grief reactions and may be at-risk for adverse grief and mental health outcomes. However, it remains unclear how those bereaved by suicide experience continuing bonds. To address this gap, we conducted a systematic review according to PRISMA guidelines. Searches of peer-reviewed literature in Medline, PsycINFO, Embase, Emcare, EBM Reviews, and Scopus identified 15 studies ( 2 quantitative and 13 qualitative) reporting on 12 samples, published between 2010 and 2021. The study quality of the quantitative studies was poor, but it was fair amongst the qualitative studies. People bereaved by suicide experienced continuing bonds across a variety of domains and reported mostly positive experiences. Factors that tended to have an impact on the expression of continuing bonds included time since bereavement, relationship to the deceased, social expectations, sex of the bereaved, and the ability of the bereaved to make meaning of the death. The review concludes that most participants reported positive experiences with continuing bonds. However, discrepancies between males and females and between those bereaved by suicide and those bereaved by other causes warrants further investigation. In addition, longitudinal community-based research involving representative samples is needed to understand the evolution and experience of continuing bonds over time in those bereaved by suicide and to inform future efforts in supporting them.
\end{abstract}

Keywords: continuing bonds; grief; bereavement; suicide; systematic review

\section{Introduction}

Suicide claims the lives of over 700,000 people per year globally [1]. A recent metaanalysis of population-based studies indicated that approximately one in twenty people experience a loss by suicide in one given year and one in five do so during their lifetime, although the impact of the death may depend on the closeness of the relationship [2]. Due to the complex nature of grief after suicide, individuals bereaved by suicide have a higher risk of adverse mental health outcomes such as posttraumatic stress disorder, depression, suicidal ideation, and attempted suicide than those bereaved by other causes [3]. People bereaved by suicide also have particular needs for professional help in dealing with their grief (for example, regarding feelings of guilt and struggles with 'why' questions) compared to those bereaved by natural causes [4].

Initially, grief literature suggested that bereaved individuals progress through different stages of grief in order to achieve detachment from the deceased person [5]. However, Klass and colleagues [6] challenged this theory and proposed the concept of continuing bonds as an alternative to detachment. They defined continuing bonds as the presence of an ongoing inner relationship with the deceased person [6]. Still, the concept of continuing bonds has been described in cultural literature and various religions over the centuries [7]. Klass and colleagues drew attention to specific rituals which demonstrated the practice of continuing bonds long before the theory gained traction in grief literature [6,7]. They gave 
the example of Japanese Buddhism, in which the deceased become part of the spirit family and are accessible to the bereaved, and in Judaism, in which the deceased are remembered through prayer and the physical lighting of a candle [6,7].

Recent studies have aimed to characterise continuing bonds as positive or negative and to specify the ways in which they manifest [7]. The process of meaning-making emerged as an important tool in the formation of positive bonds [8]. Meaning-making refers to the way in which bereaved individuals process the death in a way that holds meaning for them [9]. The concept of continuing bonds has gained traction over the years, as witnessed through the development of designated instruments [10] and studies in various bereaved populations (such as after the death of a parent or a child) [7]. However, it remains unclear how continuing bonds manifest themselves in those bereaved by suicide and how this population experiences continuing bonds. To-date, no review on this topic has been conducted. Still, a better understanding of this aspect of grief in people bereaved by suicide may provide useful information for future efforts in supporting them.

This review will address this gap by synthesising and analysing the research on continuing bonds in individuals bereaved by suicide. It aims to characterise aspects of the continuing bond including how they manifest, and whether those bereaved experience them as positive or negative.

\section{Materials and Methods}

\subsection{Search Strategy}

The review adhered to PRISMA guidelines [11], and the protocol was registered in the PROSPERO database (CRD42021271971). The review involved systematic searches of the following databases: Medline, PsycINFO, Embase, Emcare and EBM Reviews (all accessed via Ovid), and Scopus. The search in Medline comprised MeSH and text words: (Continu* bond*.mp OR continu* ${ }^{*}$ relationship*.mp OR continu* connection*.mp OR Ongoing bond*.mp OR Ongoing relationship*.mp OR Ongoing connection*.mp OR meaning making.mp OR Memorial*.mp OR Ritual*.mp) AND (Grie*.mp OR Grief/ OR Mourn*.mp OR Bereav*.mp OR Bereavement/ OR Suicide bereave*.mp OR Bereave* by suicide.mp OR loss by suicide.mp OR suicide loss survivor*.mp) AND (suicide.mp OR Suicide/). A similar search string including headings and keywords was used in the other databases.

One researcher (R.G.) conducted the search in August 2021. It was limited to publications in English but not by date of publication. Two researchers (R.G., K.A.) assessed titles and abstracts for eligibility and any discordance was resolved through discussion with the third researcher (K.K.). The same two researchers then assessed the full text of potentially relevant studies using the inclusion/exclusion criteria. Researcher R.G. hand-searched the references of the included studies and conducted a forward-citation search in Google Scholar to identify any further studies. Figure 1 summarises the search strategy.

\subsection{Inclusion and Exclusion Criteria}

Studies were included if: (1) the study population consisted of people bereaved by suicide; (2) the study provided empirical qualitative and quantitative data on continuing bonds with an individual lost to suicide; (3) the study was published in the English language; and (4) the study was published as a paper in a peer-reviewed journal.

The review excluded: (1) studies not providing data specifically on people bereaved by suicide; (2) studies not providing data on continuing bonds in the context of suicide bereavement; (3) studies based on other methods, such as case studies, reviews and opinion paper; (4) studies not written in English; and (5) studies which were not peer-reviewed. Unclarity on the inclusion of three studies was resolved through discussion with the third researcher (K.K.). 


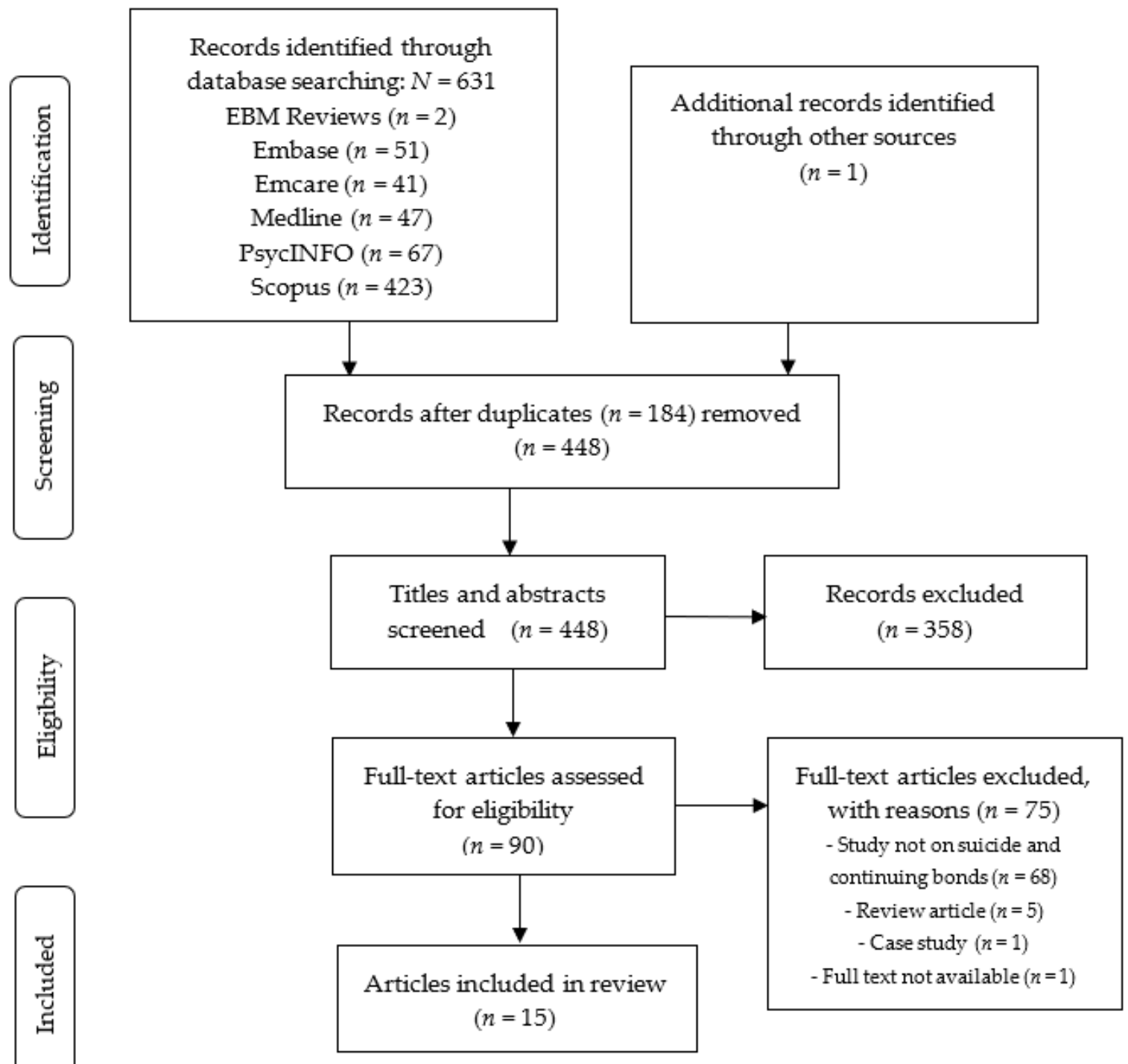

Figure 1. PRISMA Flow Diagram.

\subsection{Data Extraction}

Two researchers (R.G., K.A.) independently extracted the following data: author, year and location of study, eligibility criteria, sample size, participants' sex, age, time since bereavement and relationship to the deceased, setting, study design, and main findings.

\subsection{Quality Assessment}

Two researchers (R.G., K.A.) independently conducted the quality assessment and resolved disagreements through discussion. No eligible study was excluded based on its quality. Quantitative studies were assessed using the Newcastle-Ottawa Quality Assessment Form for Cohort Studies [12], comprising eight items across three domains: (1) selection (four items), (2) comparability (one item), and (3) outcome (three items). Scores in each domain were totalled to determine study quality as good, fair, or poor. The interrater reliability was high $(\kappa=0.82)$.

The qualitative studies were assessed using the Consolidated Criteria for Reporting Qualitative Research (COREQ) [13] consisting of thirty-two items across three domains: (1) research team and reflexivity (eight items), (2) study design (fifteen items), and (3) analysis and findings (nine items). For each study, the number and percentage of items satisfied 
within each domain and across all domains was calculated. The interrater agreement was high $(\kappa=0.92)$.

\section{Results}

\subsection{Study Characteristics}

The searches identified fifteen studies reporting on twelve samples. Five studies were conducted in Australia [14-18], three in the USA $[9,19,20]$, three in the UK [21-23], two in Israel [24,25], one in Canada [26], and one in Switzerland [27]. There were two quantitative and thirteen qualitative studies. The quantitative studies collected data via online questionnaires $[19,24]$. One also utilised the Two-Track Bereavement Questionnaire (TTBQ) [10] to measure the level of functioning of bereaved individuals and their relationship with the deceased person [24]. Eleven of the thirteen qualitative studies involved semi-structured or in-depth interviews either in-person or via telephone, one utilised a survey [20] and one collected data through a series of workshops over twelve weeks [18].

The quantitative studies had sample sizes ranging from $N=159$ [24] to $N=1301$ [19], whilst qualitative studies had sample sizes ranging from $N=7$ [14] to $N=50$ [27], with the exception of Jahn $[19,20]$ who used the same data set for both a quantitative and qualitative study. Tables 1 and 2 summarise the quantitative and qualitative studies, respectively.

A large percentage (67-89\%) of participants in most studies $(n=9)$ were female [16, 19-22,24-27]. Three studies had equal numbers of both sexes [15,17,23], two had slightly more male $(57-60 \%)$ than female participants $[9,14]$, and one study did not report the sex of participants [18]. Across studies, the age range was between 8 and 85 years [19,23].

Whilst most studies included a broad range of relationships between the bereaved individuals and the deceased, six focused on a specific relationship, including bereaved parents $(n=3)$ [15-17], siblings $(n=2)$ [14,25], and children $(n=1)$ [23].

Time since death ranged between two months and over forty-five years $[18,24]$. Two studies focused on the grief reactions from five months onwards [21,22], whilst others looked at continuing bonds and grief reactions on a longer-term from five years onward [9] or within a ten-year period [14]. Eight studies did not limit participants by time since loss $[16,19,20,23-27]$, and three included longitudinal data $[15,17,18]$.

\subsection{Quality Assessment}

Appendix A presents the methodological quality of the quantitative studies, and the two included studies received a rating of 'poor' quality. One study [24] scored well in the 'comparability' domain, but both studies $[19,24]$ tended to score poorly in the 'selection' and 'outcome' domains by using selected samples and relying on self-reported outcomes. Appendix B outlines the quality assessment of the 13 qualitative studies. The studies satisfied between 34\% [22] and 75\% [15,17] of the COREQ criteria [13]. Most studies reported only few items across the 'research team and reflexivity' domain (on average 35\% of items were reported). However, most items (on average 63\%) in both the 'study design' and 'analysis and findings' domains were reported.

\subsection{Study Findings}

\subsubsection{In What Way Can Continuing Bonds Manifest?}

Findings from the included studies showed that continuing bonds manifested or were experienced in various ways. Quantitative data showed that $63 \%$ of participants reported having spiritual experiences [19]. The most common experiences included dreams about the deceased (73.4\%), feeling the presence of the deceased (53\%), and profound coincidences (40.3\%) [19]. In qualitative studies, participants reported the use of rituals and memorials (both physical and online) $[9,16,21,23]$, spiritual and religious beliefs $[9,26,27]$, metaphysical experiences [20,26], physical objects [9], and suicide-related thoughts and behaviours, such as a desire to join the deceased and/or understand what they went through [9]. 
Table 1. Summary of quantitative studies.

\begin{tabular}{|c|c|c|c|c|c|c|c|c|c|c|}
\hline $\begin{array}{l}\text { Author, Year, } \\
\text { Location }\end{array}$ & $\begin{array}{c}\text { Eligibility } \\
\text { Criteria }\end{array}$ & Sample Size & Male/Female & Age & $\begin{array}{c}\text { Time since } \\
\text { Bereavement }\end{array}$ & $\begin{array}{l}\text { Relationship to } \\
\text { Deceased }\end{array}$ & Setting & Study Design & $\begin{array}{l}\text { Outcome } \\
\text { Measures }\end{array}$ & Main Results \\
\hline $\begin{array}{l}\text { Levi-Belz (2017) } \\
\text { Israel [24] }\end{array}$ & $\begin{array}{l}>18 \mathrm{y} / \mathrm{o} \\
\text { identify as } \\
\text { a bereaved } \\
\text { family member. } \\
\text { Excl.: }<15 \mathrm{y} / \mathrm{o} \\
\text { at time of death, } \\
\text { inability to } \\
\text { speak or write } \\
\text { in Hebrew } \\
\text { or English. }\end{array}$ & $\begin{array}{c}N=159 \text { suicide } \\
\text { loss survivors }=58, \\
\text { sudden-death } \\
\text { bereavers }=48 \\
\text { expected-death } \\
\text { bereavers }=53 \\
(\mathrm{~F}=81 \%)\end{array}$ & $\begin{array}{c}\mathrm{M}=30 \\
\mathrm{~F}=129 \\
\text { suicide loss } \\
\text { survivors: } \\
\mathrm{M}=13, \mathrm{~F}=45 \\
\text { sudden-death } \\
\text { bereavers: } \\
\mathrm{M}=6, \mathrm{~F}=42 \\
\text { expected-death } \\
\text { bereavers: } \\
\mathrm{M}=11, \mathrm{~F}=42\end{array}$ & $\begin{array}{c}18-73 \text { suicide } \\
\text { loss survivor } \\
\mathrm{M}=40.5, \mathrm{SD}=15.6 \\
\text { sudden-death } \\
\text { bereavers } \\
\mathrm{M}=35.7 \\
\mathrm{SD}=13.1 \\
\text { expected-death } \\
\text { bereavers } \\
\mathrm{M}=33.1, \mathrm{SD}=12.3\end{array}$ & $\begin{array}{c}2 \mathrm{~m}-45 \mathrm{y} \\
(\mathrm{M}=95 \mathrm{~m} \\
\mathrm{SD}=105.46 \mathrm{~m})\end{array}$ & $\begin{array}{c}25 \text { parents, } \\
11 \text { children, } \\
26 \text { siblings, } \\
30 \text { spouses, } \\
\text { and } 44 \text { close } \\
\text { family members, } \\
9 \text { unknown }\end{array}$ & $\begin{array}{c}\text { Online } \\
\text { questionnaire }\end{array}$ & Cross sectional & $\begin{array}{c}\text { Level of } \\
\text { functioning: } \\
\text { Two-track } \\
\text { bereavement } \\
\text { questionnaire } \\
\text { (TTBQ), } \\
\text { Post-traumatic } \\
\text { grief level: } \\
\text { Stress-Related } \\
\text { Growth Scale } \\
\text { (SRGS) }\end{array}$ & $\begin{array}{l}\text { Suicide-loss survivors } \\
\text { reported lower levels of } \\
\text { close positive continuing } \\
\text { bonds with the deceased than } \\
\text { did participants from the } \\
\text { sudden- and expected-death } \\
\text { bereaved groups. } \\
\text { Intense grief and } \\
\text { preoccupation were } \\
\text { associated with less positive } \\
\text { personal transformation, } \\
\text { particularly among the suicide } \\
\text { bereaved. This suggests that } \\
\text { continuing bonds in those } \\
\text { bereaved by suicide are more } \\
\text { likely to be experienced } \\
\text { negatively than in those } \\
\text { bereaved by other causes. }\end{array}$ \\
\hline $\begin{array}{c}\text { Jahn et al. (2014) } \\
\text { USA [19] }\end{array}$ & $\begin{array}{l}\text { Survivors of } \\
\text { suicide loss }\end{array}$ & $N=1301$ & $\begin{array}{c}\mathrm{M}=135 \\
\mathrm{~F}=1155 \\
\mathrm{U}=11\end{array}$ & $40-85$ & $\begin{array}{c}1 \mathrm{y}-3 \mathrm{y} \\
(28.9 \% ; n=376), \\
<1 \mathrm{y} \\
(25.7 \% ; n=334), \\
4 \mathrm{y}-10 \mathrm{y} \\
(22.0 \% ; n=286), \\
11 \mathrm{y}-20 \mathrm{y} \\
(12.2 \% ; n=159), \\
\text { and }>20 \mathrm{y} \\
(8.2 \% ; n=107) .\end{array}$ & $\begin{array}{c}\text { Parent }(n=362), \\
\text { sibling }(n=235), \\
\text { spouse/partner } \\
(n=204), \\
\text { child }(n=180), \\
\text { friend }(n=112), \\
\text { niece/nephew } \\
(n=21), \\
\text { grandchild } \\
(n=9), \text { another } \\
\text { relationship } \\
(n=119) .\end{array}$ & $\begin{array}{c}\text { Two } \\
\text { questionnaires- } \\
\text { one demographics } \\
\text { and one about } \\
\text { experience of } \\
\text { suicide. Both } \\
\text { containing both } \\
\text { qualitative and } \\
\text { quantitative info }\end{array}$ & $\begin{array}{l}\text { Descriptive } \\
\text { statistics used to } \\
\text { explore } \\
\text { characteristics } \\
\text { of loss and chi } \\
\text { squared to } \\
\text { examine } \\
\text { demographical } \\
\text { differences } \\
\text { between } \\
\text { responses }\end{array}$ & $\begin{array}{l}\text { Two questionnaires: } \\
\text { (i) demographic } \\
\text { questionnaire, } \\
\text { (ii) spiritual } \\
\text { experiences of } \\
\text { survivors of } \\
\text { suicide, including } \\
\text { questions about } \\
\text { type and } \\
\text { frequency of } \\
\text { spiritual } \\
\text { experiences }\end{array}$ & $\begin{array}{c}\text { Most }(n=819,63 \%) \text { of those } \\
\text { bereaved by suicide reported } \\
\text { after-death spiritual } \\
\text { experiences including dreams, } \\
\text { feeling the presence of } \\
\text { the person and } \\
\text { profound coincidences. } \\
\text { These experiences are often } \\
\text { regarded as positive with } \\
74.5 \% \text { of participants }(n=610) \\
\text { finding them 'helpful' and } \\
\text { only } 4.8 \% \text { ( } n=39 \text { ) viewing } \\
\text { them as 'harmful' (19.9\%, } \\
n=163 \text { saw them as neither } \\
\text { helpful nor harmful). } \\
\text { Being female and having a } \\
\text { closer pre death relationship } \\
\text { (family member as opposed to } \\
\text { friend or client) was positively } \\
\text { associated with } \\
\text { spiritual experiences. }\end{array}$ \\
\hline
\end{tabular}


Table 2. Summary of qualitative studies.

\begin{tabular}{|c|c|c|c|c|c|c|c|c|c|}
\hline $\begin{array}{l}\text { Author, Year, } \\
\text { Location }\end{array}$ & Eligibility Criteria & Sample Size & Male/Female & Age (Years) & $\begin{array}{c}\text { Time Since } \\
\text { Bereavement }\end{array}$ & $\begin{array}{c}\text { Relationship to } \\
\text { Deceased }\end{array}$ & Setting & Study Design & Main Results \\
\hline $\begin{array}{l}\text { Adams et al. } \\
\text { (2019) AUS [14] }\end{array}$ & $\begin{array}{c}\text { Bereaved by } \\
\text { suicide of sibling } \\
\text { (sibling <20 y/o) } \\
\text { in past } 10 \mathrm{yrs}\end{array}$ & $N=7$ & $\begin{array}{l}M=4 \\
F=3\end{array}$ & $\begin{array}{c}20-27 \text { at time of } \\
\text { interview (16-23 } \\
\text { at time of suicide) }\end{array}$ & Average time $=3$ y $9 \mathrm{~m}$ & Sibling & Telephone interview & $\begin{array}{c}\text { Interpretive } \\
\text { phenomen-ological } \\
\text { analysis (IPA) }\end{array}$ & $\begin{array}{l}\text { Identified } 4 \text { main themes: } \\
\text { (a) the process of grief, } \\
\text { (b) grief interactions } \\
\text { (within families and outside), } \\
\text { (c) continuing bonds, and } \\
\text { (d) meaning-making and } \\
\text { growth through grief. }\end{array}$ \\
\hline $\begin{array}{l}\text { Bailey et al. } \\
\text { (2015) UK [21] }\end{array}$ & $\begin{array}{l}\text { Family members } \\
\text { and friends who } \\
\text { own suicide } \\
\text { memorial sites }\end{array}$ & $N=11$ & $\begin{array}{l}M=3 \\
F=8\end{array}$ & $20-60$ & $5 \mathrm{~m}-4 \mathrm{y}$ & $\begin{array}{l}\text { Parent, siblings } \\
\text { or friends }\end{array}$ & $\begin{array}{l}\text { Face-to-face interviews- } \\
\text { semi-structured } \\
\text { narrative style }\end{array}$ & $\begin{array}{c}\text { Qualitative } \\
\text { interpretative } \\
\text { approach, } \\
\text { combining constant } \\
\text { comparison } \\
\text { techniques with } \\
\text { thematic analysis }\end{array}$ & $\begin{array}{l}\text { The most common motivating } \\
\text { factor for starting a memorial page } \\
\text { was to 'keep the deceased alive' } \\
\text { and maintain a connection. } \\
\text { Participants found that they were } \\
\text { able to better construct and refine } \\
\text { relationships with the deceased } \\
\text { using online memorials. } \\
\text { Whilst most people had positive } \\
\text { experiences with memorial sites, } \\
\text { the dangers of becoming overly } \\
\text { attached and experiencing } \\
\text { compounding grief or 'double loss' } \\
\text { was highlighted. }\end{array}$ \\
\hline $\begin{array}{l}\text { Bell et al. } \\
\text { (2015) UK [22] }\end{array}$ & $\begin{array}{l}\text { Individuals who had } \\
\text { set up or were } \\
\text { managing memorial } \\
\text { sites for those who } \\
\text { dies by suicide }\end{array}$ & $N=11$ & $\begin{array}{l}\mathrm{M}=3 \\
\mathrm{~F}=8\end{array}$ & $20-60$ & $5 \mathrm{~m}-4 \mathrm{y}$ & $\begin{array}{l}\text { Parent, siblings } \\
\text { or friends }\end{array}$ & Individual interviews & $\begin{array}{c}\text { Qualitative } \\
\text { interpretative } \\
\text { approach, } \\
\text { combining constant } \\
\text { comparison } \\
\text { techniques with } \\
\text { thematic analysis }\end{array}$ & $\begin{array}{l}\text { Provided insight into how online } \\
\text { memorialisation allows more } \\
\text { flexibility and depth in the } \\
\text { exploration of grief than can } \\
\text { traditional mourning objects-this } \\
\text { allows users to reminisce on } \\
\text { positive aspects of deceased life } \\
\text { but can elicit negative experiences } \\
\text { as users can't control how } \\
\text { memories are framed. }\end{array}$ \\
\hline $\begin{array}{c}\text { Castelli } \\
\text { Dransart (2018) } \\
\text { Switzerland [27] }\end{array}$ & $\begin{array}{l}\text { Suicide-survivors: } \\
\text { A person was } \\
\text { considered as a } \\
\text { survivor of suicide } \\
\text { if: (1) he/she } \\
\text { self-qualified as } \\
\text { such; (2) he/she felt } \\
\text { emotionally close to } \\
\text { the deceased; and } \\
\text { (3) his/her life had } \\
\text { been disrupted } \\
\text { by a suicide } \\
\text { (self-perception). } \\
\text { >18 y/o, able to } \\
\text { speak Italian, French } \\
\text { or German }\end{array}$ & $N=50$ & $\begin{array}{l}\mathrm{M}=11 \\
\mathrm{~F}=39\end{array}$ & $14-73$ & $<12 \mathrm{~m}-16 \mathrm{y}$ & $\begin{array}{c}18 \text { mothers, } \\
5 \text { fathers, } \\
10 \text { sisters, } \\
3 \text { brothers, } \\
3 \text { daughters, } \\
1 \text { son, } 7 \text { partners, } \\
1 \text { aunt, } 2 \text { friends }\end{array}$ & $\begin{array}{l}\text { Face-to-face in depth } \\
\text { interviews conducted by } \\
\text { author or mental health } \\
\text { carer, either at home or } \\
\text { location chosen by bereaved }\end{array}$ & $\begin{array}{l}\text { Grounded Theory } \\
\text { using constant } \\
\text { comparison of data } \\
\text { and } 3 \text { steps of } \\
\text { coding: open, } \\
\text { axial \& selective }\end{array}$ & $\begin{array}{l}\text { Suicide triggered spiritual and } \\
\text { religious thoughts and experiences } \\
\text { for most participants. Even those } \\
\text { who claimed to be atheist or } \\
\text { agnostic noted religious rituals } \\
\text { and spiritual symbols as being } \\
\text { important contributors in forging } \\
\text { and maintaining a continuing } \\
\text { bond with the deceased and in } \\
\text { honouring their memory. } \\
\text { Interviewees believed loved ones } \\
\text { continued to exist in an } \\
\text { alternative dimension or space } \\
\text { (regardless of religion). }\end{array}$ \\
\hline
\end{tabular}


Table 2. Cont.

\begin{tabular}{|c|c|c|c|c|c|c|c|c|c|}
\hline $\begin{array}{l}\text { Author, Year, } \\
\text { Location }\end{array}$ & Eligibility Criteria & Sample Size & Male/Female & Age (Years) & $\begin{array}{c}\text { Time Since } \\
\text { Bereavement }\end{array}$ & $\begin{array}{l}\text { Relationship to } \\
\text { Deceased }\end{array}$ & Setting & Study Design & Main Results \\
\hline $\begin{array}{c}\text { Entilli et al. } \\
\text { (2021) AUS [15] }\end{array}$ & $\begin{array}{l}\text { Parents who had lost } \\
\text { a child by suicide } \\
\text { less than } 6 \text { months } \\
\text { prior to starting } \\
\text { the study }\end{array}$ & $\begin{array}{l}N=14 \text { at } \\
6 \text { and } \\
12 \text { months. } \\
N=11 \text { at } \\
24 \text { months }\end{array}$ & $\begin{array}{c}\mathrm{M}=7 \\
\mathrm{~F}=7 \\
\text { (6 and } \\
12 \text { months). } \\
\mathrm{M}=6 \\
\mathrm{~F}=5 \\
\text { (at } 24 \text { months) }\end{array}$ & $\begin{array}{c}\text { Female } \\
\text { mean }=60.1 \text { years, } \\
\text { range }=50-78 \text { years } \\
\text { and male } \\
\text { mean }=59.9 \text { years, } \\
\text { range }=50-68 \text { years }\end{array}$ & $6 \mathrm{~m}, 12 \mathrm{~m}$ and $24 \mathrm{~m}$ & $\begin{array}{l}\text { Parents who had } \\
\text { lost a child (aged } \\
15-51 \text { ) by suicide. } \\
\text { Ten were bereaved } \\
\text { of sons and four } \\
\text { bereaved of } \\
\text { daughters }\end{array}$ & $\begin{array}{l}\text { Semi-structured interviews } \\
\text { either phone or face-to-face }\end{array}$ & $\begin{array}{l}\text { Longitudinal study } \\
\text { using thematic } \\
\text { analysis }\end{array}$ & $\begin{array}{l}\text { Three key themes were identified } \\
\text { in an earlier analysis (at } 6 \text { and } \\
12 \text { months post loss): searching for } \\
\text { answers and sense-making, coping } \\
\text { strategies and support, and finding } \\
\text { meaning and purpose. } \\
\text { Further exploration of these } \\
\text { themes at } 24 \text { months revealed } \\
\text { significant differences between } \\
\text { mothers and fathers with the latter } \\
\text { adopting more maladaptive } \\
\text { coping strategies. } \\
\text { Maintaining the relationship with } \\
\text { the deceased and paranormal } \\
\text { experiences were new themes at } \\
24 \text { months (not present at } 6 \text { and } \\
12 \text { months) and a shift from } \\
\text { brooding to reflection/sense- } \\
\text { making was seen at } 24 \text { months. } \\
\text { The adaptation process was } \\
\text { fluctuating and dynamic. }\end{array}$ \\
\hline $\begin{array}{l}\text { Gall et al. (2015) } \\
\text { Canada [26] }\end{array}$ & $\begin{array}{l}\text { Individuals who had } \\
\text { personal experiences } \\
\text { of suicide } \\
\text { bereavement }\end{array}$ & $\begin{array}{c}\quad N=15 \\
\text { (11 bereaved } \\
\text { and } 4 \text { mental } \\
\text { health workers) }\end{array}$ & $\begin{array}{c}\mathrm{M}=2 \\
\mathrm{~F}=9 \\
\text { (bereaved) } \\
\mathrm{M}=1 \\
\mathrm{~F}=3 \\
(\mathrm{MHW})\end{array}$ & $\begin{array}{l}\text { Mean age was } \\
49 \text { (bereaved) } \\
\text { and } 53(\mathrm{MHW})\end{array}$ & $\begin{array}{c}\text { Mean }=13 \mathrm{y} \\
\text { minimum of } 2 y\end{array}$ & $\begin{array}{l}\text { The deceased } \\
\text { persons were: } \\
\text { four sons, } \\
\text { two fathers, } \\
\text { two close friends, } \\
\text { two uncles and } \\
\text { one mother. }\end{array}$ & Semi-structured interviews & $\begin{array}{c}\text { Phenomenological } \\
\text { approach, } \\
\text { thematic analysis }\end{array}$ & $\begin{array}{l}\text { Individuals had difficulty } \\
\text { reconciling the suicide death of } \\
\text { a loved one with their religious } \\
\text { views. This often led to } \\
\text { a personally defined spirituality } \\
\text { rather than a complete loss of faith. } \\
\text { Many individuals (also } \\
\text { non-religious) found meaning in } \\
\text { the belief of an afterlife and/or felt } \\
\text { hopeful that the deceased was in } \\
\text { a better place and would someday } \\
\text { reconnect with them. Engagement } \\
\text { in activities to maintain a bond } \\
\text { were common. }\end{array}$ \\
\hline \multirow[t]{2}{*}{$\begin{array}{l}\text { Hunt et al. } \\
\text { (2019) USA [9] }\end{array}$} & \multirow[t]{2}{*}{$\begin{array}{c}>18 \mathrm{y} / \mathrm{o} \\
\text { self-identified as } \\
\text { suicide loss survivor, } \\
>5 \text { years since death }\end{array}$} & \multirow[t]{2}{*}{$N=10$} & \multirow[t]{2}{*}{$\begin{array}{l}\mathrm{M}=6 \\
\mathrm{~F}=4\end{array}$} & \multirow[t]{2}{*}{$\begin{array}{c}30-72 \\
(\text { mean }=47.6)\end{array}$} & \multirow[t]{2}{*}{$\begin{array}{c}5 y-30 y \\
(m e a n=18.6 y)\end{array}$} & \multirow[t]{2}{*}{$\begin{array}{l}5 \text { siblings, } \\
2 \text { partners, } \\
2 \text { parents and } \\
\text { one participant } \\
\text { who had lost } \\
\text { a sibling, } \\
\text { grandfather } \\
\text { and cousin }\end{array}$} & \multirow[t]{2}{*}{$\begin{array}{c}\text { Face-to-face } \\
\text { semi-structured interviews } \\
\text { at participants homes }\end{array}$} & \multirow[t]{2}{*}{$\begin{array}{l}\text { Thematic analysis } \\
\text { informed by } \\
\text { grounded theory }\end{array}$} & $\begin{array}{l}\text { Three major themes } \\
\text { were identified: } \\
\text { one harmful (feelings of } \\
\text { responsibility) } \\
\text { one helpful (making mean- } \\
\text { ing) the the process of suicide } \\
\text { bereavement. } \\
\text { one that helped shift } \\
\text { from harmful to helpful } \\
\text { (social support) }\end{array}$ \\
\hline & & & & & & & & & $\begin{array}{l}\text { Participants did not identify } \\
\text { with the Kubler-Ross } \\
\text { stages of grief theory. }\end{array}$ \\
\hline
\end{tabular}


Table 2. Cont.

\begin{tabular}{|c|c|c|c|c|c|c|c|c|c|}
\hline $\begin{array}{l}\text { Author, Year, } \\
\text { Location }\end{array}$ & Eligibility Criteria & Sample Size & Male/Female & Age (Years) & $\begin{array}{c}\text { Time Since } \\
\text { Bereavement }\end{array}$ & $\begin{array}{l}\text { Relationship to } \\
\text { Deceased }\end{array}$ & Setting & Study Design & Main Results \\
\hline $\begin{array}{l}\text { Jahn et al. } \\
\text { (2018) USA [20] }\end{array}$ & $\begin{array}{l}\text { Any person who } \\
\text { identified as } \\
\text { bereaved by suicide }\end{array}$ & $N=1301$ & $\begin{array}{c}\mathrm{M}=135 \\
\mathrm{~F}=1155 \\
\mathrm{U}=11\end{array}$ & $40-85$ & $\begin{array}{c}1 \mathrm{y}-3 \mathrm{y} \\
(28.9 \% ; n=376), \\
<1 \mathrm{y}(25.7 \% ; n=334), \\
4 \mathrm{y}-10 \mathrm{y} \\
(22.0 \% ; n=286), \\
11 \mathrm{y}-20 \mathrm{y} \\
(12.2 \% ; n=159), \text { and } \\
>20 \mathrm{y}(8.2 \% ; n=107) .\end{array}$ & $\begin{array}{l}\text { Anyone bereaved } \\
\text { by suicide }\end{array}$ & $\begin{array}{l}\text { Two questionnaires-one } \\
\text { demographics and one } \\
\text { about spiritual experiences } \\
\text { after suicide bereavement. } \\
\text { Both containing both } \\
\text { qualitative and } \\
\text { quantitative info }\end{array}$ & $\begin{array}{l}\text { Inductive thematic } \\
\text { analysis }\end{array}$ & $\begin{array}{l}\text { Nine main themes were identified: } \\
\text { (1) a helpful sense of comfort; } \\
\text { (2) a helpful sense of connection } \\
\text { with the deceased; (3) intense } \\
\text { sadness evoked by the spiritual } \\
\text { experiences; (4) confusion } \\
\text { regarding the spiritual experiences, } \\
\text { (5) negative reminders of the } \\
\text { deceased or negative meanings of } \\
\text { spiritual experiences; (6) evidence } \\
\text { of an afterlife; (7) general } \\
\text { importance of the spiritual } \\
\text { experiences' meaning; (8) impact } \\
\text { of and on religious beliefs; and } \\
\text { (9) others' responses to disclosure } \\
\text { of suicide or spiritual experiences. } \\
\text { Generally, participants found } \\
\text { spiritual experiences aided in } \\
\text { healing and transformation and } \\
\text { were regarded as positive. }\end{array}$ \\
\hline $\begin{array}{l}\text { Leichtentritt } \\
\text { et al. (2015) } \\
\text { Israel [25] }\end{array}$ & $\begin{array}{l}\text { Having experienced } \\
\text { the loss of a sibling } \\
\text { to suicide and the } \\
\text { death having } \\
\text { occurred at least } \\
\text { five years prior to } \\
\text { the interview }\end{array}$ & $N=9$ & $\begin{array}{l}\mathrm{M}=3 \\
\mathrm{~F}=6\end{array}$ & $29-63$ & $5 y-37 y$ & Sibling & In-depth interviews & $\begin{array}{l}\text { Relational dialect } \\
\text { theory and narrative } \\
\text { analysis used. }\end{array}$ & $\begin{array}{l}\text { Five characteristics of the post } \\
\text { death relationship were identified, } \\
\text { each existing along a continuum: } \\
\text { (1) concrete-symbolic } \\
\text { (2) dynamic-static } \\
\text { (3) conscious-unconscious } \\
\text { (4) personal-public } \\
\text { (5) monologue-dialogue } \\
\text { Findings suggest that labelling } \\
\text { post death relationships as } \\
\text { 'adaptive' or 'maladaptive' is } \\
\text { simplistic. Bereavement can be } \\
\text { better understood when plotted } \\
\text { within the } 5 \text { continua. }\end{array}$ \\
\hline $\begin{array}{l}\text { Maple et al. } \\
\text { (2013) AUS [16] }\end{array}$ & $\begin{array}{l}\text { Parents who lost } \\
\text { a child by suicide }\end{array}$ & $N=22$ & $\begin{array}{l}M=6 \\
F=16\end{array}$ & NA & $6 \mathrm{~m} \rightarrow>26 \mathrm{y}$ & $\begin{array}{c}22 \text { parents } \\
\text { (6 fathers and } \\
16 \text { mothers) from } \\
18 \text { families } \\
\text { bereaved of } \\
15 \text { sons and } \\
3 \text { daughters. } \\
14 \text { participated } \\
\text { individually and } \\
4 \text { as couples }\end{array}$ & $\begin{array}{l}\text { In-depth interviews, mostly } \\
\text { face-to-face (one phone) }\end{array}$ & $\begin{array}{l}\text { Narrative Inquiry, } \\
\text { recursive technique } \\
\text { used to explore in } \\
\text { more depth. }\end{array}$ & $\begin{array}{l}\text { Contrary to traditional grief } \\
\text { literature, it was found that } \\
\text { parents needed to maintain } \\
\text { a relationship with their deceased } \\
\text { child. Manifestations of continuing } \\
\text { bonds varied between parents. } \\
\text { Commencing with the funeral, } \\
\text { parents began developing rituals } \\
\text { ensuring that their child's life, and } \\
\text { not the manner of death, } \\
\text { was celebrated. } \\
\text { Some participants were limited in } \\
\text { their expression of grief due to } \\
\text { social pressure to resolve grief. }\end{array}$ \\
\hline
\end{tabular}


Table 2. Cont.

\begin{tabular}{|c|c|c|c|c|c|c|c|c|c|}
\hline $\begin{array}{l}\text { Author, Year, } \\
\text { Location }\end{array}$ & Eligibility Criteria & Sample Size & Male/Female & Age (Years) & $\begin{array}{c}\text { Time Since } \\
\text { Bereavement }\end{array}$ & $\begin{array}{c}\text { Relationship to } \\
\text { Deceased }\end{array}$ & Setting & Study Design & Main Results \\
\hline $\begin{array}{c}\text { Ross et al. } \\
\text { (2018) AUS [17] }\end{array}$ & $\begin{array}{l}\text { Parents bereaved by } \\
\text { suicide loss of their } \\
\text { child } 6 \text { months prior } \\
\text { to commencement } \\
\text { of study }\end{array}$ & $N=14$ & $\begin{array}{l}\mathrm{M}=7 \\
\mathrm{~F}=7\end{array}$ & $\begin{array}{c}50-78(\text { female } \\
\text { mean }=60.1 \text { years and } \\
\text { range }=50-78, \text { male } \\
\text { mean }=59.9 \text { years and } \\
\text { range }=50-68)\end{array}$ & $6 \mathrm{~m}$ and $12 \mathrm{~m}$ & $\begin{array}{l}\text { Parents who had } \\
\text { lost a child } \\
\text { (aged 15-51) by } \\
\text { suicide. Ten were } \\
\text { bereaved of sons } \\
\text { and four bereaved } \\
\text { of daughters }\end{array}$ & $\begin{array}{l}\text { Individual, semi-structured } \\
\text { interviews either } \\
\text { face-to-face or telephone }\end{array}$ & $\begin{array}{l}\text { Longitudinal study } \\
\text { with inductive } \\
\text { qualitative } \\
\text { approach. }\end{array}$ & $\begin{array}{c}\text { Identified three key themes } \\
\text { (searching for answers and } \\
\text { sense-making, coping strategies } \\
\text { and support, and finding meaning } \\
\text { and purpose) in parental response } \\
\text { to suicide bereavement. } \\
\text { The phases of sense-making and } \\
\text { meaning-making experienced by } \\
\text { participants and the range of both } \\
\text { adaptive and maladaptive coping } \\
\text { strategies indicated that adapting } \\
\text { to bereavement is a dynamic and } \\
\text { fluctuating process. }\end{array}$ \\
\hline $\begin{array}{c}\text { Sands et al. } \\
\text { (2010) AUS [18] }\end{array}$ & $\begin{array}{l}\text { Adults }>19 \mathrm{y} / \mathrm{o}, \\
\text { bereaved through } \\
\text { the suicide death of } \\
\text { a significant person } \\
\text { in their lives }\end{array}$ & $N=16$ & NA & Aged 19+ & $>2 \mathrm{~m}$ & $\begin{array}{l}\text { Family members } \\
\text { or spouses: } \\
\text { partner grieving } \\
\text { a partner, parent } \\
\text { grieving a child, } \\
\text { sibling grieving } \\
\text { a sibling, and } \\
\text { adult child } \\
\text { grieving a parent }\end{array}$ & $\begin{array}{l}\text { A series of workshops } \\
\text { delivered over } 12 \text { weeks } \\
\text { and } 30 \text { h-involved } \\
\text { discussion, artwork, grief } \\
\text { rituals and journal writing }\end{array}$ & $\begin{array}{l}\text { Generic thematic } \\
\text { analysis }\end{array}$ & $\begin{array}{l}\text { Identified } 3 \text { core themes that } \\
\text { assisted in meaning making in } \\
\text { relationships with the themselves, } \\
\text { the deceased, and with others. } \\
\text { The } 3 \text { themes were: (i) } \\
\text { Intentionality ('tying on the shoes } \\
\text { or adopting the perspective of the } \\
\text { deceased), (ii) Reconstruction } \\
\text { ('walking in the shoes'), } \\
\text { (iii) Repositioning ('taking off } \\
\text { the shoes') }\end{array}$ \\
\hline
\end{tabular}


Suicide bereaved individuals tended to experience continuing bonds across multiple domains both concurrently and over time $[15,25]$. It was common to experience continuing bonds across a continuum, for example, engaging in both public and private expressions of continuing bonds [25]. A public manifestation may include participation in ceremonies or rituals such as a funeral whilst a private manifestation may include writing to or talking to the deceased [25]. Although the process of developing continuing bonds was never described as linear, time since bereavement did appear to affect how the bonds were experienced. Entilli and colleagues [15] and Ross and colleagues [17] described experiences with continuing bonds as they appeared at 6, 12, and 24 months, observing that memorialisation and intrusive thoughts were present early on whereas paranormal beliefs and the maintenance of the relationship with the deceased were new themes at 24 months. Similarly, Sands and colleagues [18] reported themes as they appeared during workshops spanning over 12 weeks. Participants experienced thoughts around 'why' the person had died by suicide before reconstructing the death story and repositioning the relationship in order to establish positive ongoing bonds [18]. Suicidal ideation with a desire to join the deceased and/or to understand what they went through was another common initial reaction that typically subsided over time $[9,14]$.

Qualitative studies found that most participants wanted to experience continuing bonds and actively pursued them $[14,26]$. They achieved this through the creation of online memorials [21,22], visiting places that were frequented by the deceased [16], keeping objects that belonged to the deceased [26], actively reminiscing about the deceased [26], and writing to the deceased [15]. However, some participants found that the social expectations of grief and the stigma associated with suicide limited their public expression of continuing bonds, forcing them to express their grief privately $[16,21,22]$.

There were differences in the types of bonds experienced according to sex and type of relationship to the deceased person $[15,20,21]$. A qualitative study found that female participants and those who had a close pre-death relationship with the deceased family member or partner had more spiritual experiences with the deceased [20]. Females also created more online memorials than males [21]. Conversely, males appeared to engage more in avoidant coping strategies and would express their grief less openly [15]. Though children may experience continuing bonds in similar domains as adults, research suggests they tend to interpret their experiences differently and this varies depending on age [23].

\subsubsection{Continuing Bonds as a Positive or Negative Experience}

Participants generally perceived continuing bonds as positive experiences [16,17,19-21,27], which they associated with comfort and hope [26]. Although less common, negative experiences were those that were beyond the control of the bereaved individuals and included subconscious thoughts or feelings [25], spiritual or metaphysical experiences [26], or the unexpected discovery of facts relating to the deceased that were not in-line with the perceptions of the bereaved [21]. A quantitative study [19] demonstrated that $74.5 \%$ of participants interpreted spiritual experiences with the deceased as helpful whilst only $4.8 \%$ viewed them as harmful [19]. However, another quantitative study comparing outcomes of those bereaved by suicide with those bereaved by sudden or expected death found that those bereaved by suicide had lower levels of close positive relationships with the deceased both pre- and post-death, including less expression of continuing bonds [24].

\section{Discussion}

This review aimed to synthesise the research literature concerning continuing bonds in people bereaved by suicide, including how they manifest and whether the bereaved experienced them as positive or negative. The review identified 15 studies reporting on 12 samples. Factors that affected the way in which continuing bonds manifested included time since death, type of relationship, societal expectations, sex of the bereaved person, and their ability to make meaning of the death. In all but one study [22], participants reported mostly positive thoughts, feelings and experiences regarding continuing bonds. 


\subsection{Factors That Affect the Manifestation of Continuing Bonds}

Aside from two longitudinal studies [15,17], there appears to be little research on the effect of time since bereavement on the manifestation of continuing bonds in those bereaved by suicide. Participants from one study [9] reported that the intensity of the bonds had not lessened up to 10 years following the suicide. This is supported by a quantitative study [24] which found that when compared to those bereaved by sudden or expected deaths, those bereaved by suicide tended to maintain more intense bonds for a longer period of time and consequently took longer to engage in 'meaning-making'. These findings are important due to the strong association between intense grief and less positive personal transformation [24], highlighting the need for more longitudinal, comparative data.

Societal beliefs and expectations may impact on the manifestation of continuing bonds in several ways $[16,21,22,27]$. Some participants felt they were forced to express their ongoing relationship more privately due to the stigma surrounding suicide $[9,21]$, and the expectation to resolve grief [16]. Our review revealed that suicidal ideation $[9,14]$ in suicide bereaved individuals can be a way to reconnect with the deceased or to understand what they went through, suggesting that suicidal ideation may be a manifestation of continuing bonds in those bereaved by suicide. Interestingly, participants claimed that this process aided their grief work by helping them to make sense of the suicide; however, reported they felt discouraged from sharing these thoughts with others [9]. These findings illustrate the importance of addressing suicidal thoughts in a constructive manner as opposed to inadvertently reinforcing suicide stigma by silencing them [9].

The stigma associated with suicide in the context of religions may be particularly strong, with some bereaved individuals choosing to hold private ceremonies following a suicide death $[27,28]$. This is supported by a qualitative systematic review that found that religious individuals often isolate themselves due to feelings of shame and suicide-related stigmatisation emanating from religious doctrines [29]. Interestingly, taking part in religious ceremonies was common practice even in those participants who identified as agnostic [27]. A possible explanation for this may be that societal and cultural expectations dictate how we grieve, insofar as rituals such as funerals are expected in western culture [30]. Future studies conducted in different cultures may shed light on the expression of continuing bonds with regards to spiritual and religious themes and the possible stigma in this context.

Referring to the 'Dual Process Model of Coping with Bereavement' [31], studies reported differences between males and females with females engaging in more 'lossoriented' behaviours, including continuing bonds, and males engaging in more 'restorationoriented' behaviours, including learning new skills [15]. Whilst it may be the case that males experience continuing bonds to a lesser extent than females and/or in different domains [32], literature suggests that societal perceptions of gender and masculinity may influence the expression of grief, and grief after suicide, in males [33], resulting in males engaging in restoration-oriented activities as a distraction from or expression of their grief [33]. This view is supported by Entilli and colleagues [15] who found that fathers avoided discussing their feelings regarding the loss by suicide. Future studies may further compare the experience of continuing bonds between males and females and inform services to be directed accordingly.

The process of 'meaning-making' was mentioned throughout the reviewed studies. It refers to the ability of the bereaved person to make sense of or find meaning in the death $[8,34]$ and has been widely recognised in grief literature [8,34]. Milman and colleagues described meaning-making as a method of alleviating the cognitive dissonance caused by the death through purposeful reflection [34]. They highlight the importance of differentiating this process from rumination as the latter fails to negotiate this discrepancy and is characterised by passive, repetitive and negative thoughts [34]. Our review indicated that those who were able to make meaning of the suicide experienced more positive continuing bonds whilst those who were unable to make meaning experienced more negative continuing bonds. In addition, high resilience characteristics and a strong social support network tended to predict less intense continuing bonds and higher levels of posttraumatic growth [24]. 


\subsection{Continuing Bonds as a Positive or a Negative Experience}

Most participants in the included studies reported positive feelings experienced with continuing bonds $[16,18,23]$, a finding that is mirrored by those of general bereavement studies $[35,36]$. However, a quantitative study comparing different forms of bereavement indicated that those bereaved by suicide reporting lower levels of close and positive relationships with the deceased both pre-and post-death [24]. The incorporation of multiple factors, including close and positive, and pre-and post-death relationships, make it hard to accurately compare these results with other studies. As demonstrated by Leichtentritt and colleagues [25], a 'close' relationship is not necessarily synonymous with a 'positive' one. Likewise, as suggested throughout this review, the process of meaning-making transforms the relationship so that the pre- and post-death relationships may not be equivalent. Further studies comparing experiences of continuing bonds between those bereaved by different causes may further clarify how to understand these experiences.

Negative experiences occurred when continuing bonds in the form of memories or objects faded or were lost [14,21], when participants were not able to make meaning of the loss [15], or when aspects of continuing bonds were beyond the control of the bereaved $[22,25]$. Participants experienced feelings of distress at fading memories of the deceased [14], or due to the sudden and unexpected disappearance of online memorials [21], which was described as a 'double loss' [22]. Some expressed apprehension and guilt as contributing to the continuing bonds as they did not want to abandon the deceased person [10]. Additionally, participants experienced cognitive dissonance when their religious beliefs were in opposition to the actions of the deceased [27], or upon the discovery of new information about the deceased that was contradictory to their own internal representation of the deceased person [21,22]. These psychological discrepancies were found to hinder the ability of the bereaved person to make meaning of the death and enhanced negative feelings associated with continuing bonds [9]. Further research may clarify in which circumstances it may be more beneficial for the bereaved person to relinquish than to maintain the bond $[35,36]$.

Participants utilised several tools and behaviours to create or maintain positive continuing bonds. Many studies observed the tendency of participants to omit certain aspects of the deceased personality or behaviour when recalling memories of them [23], or to hold more symbolic or fictitious representations of the deceased $[25,27]$. Some bereaved individuals favoured online memorials due to them being more interactive than traditional mourning objects [21]. This is supported by literature reporting that this allows the bereaved to explore the relationship in more depth and to discover new things about the deceased, thus enabling the relationship to evolve [37]. Nonetheless, a potential negative aspect of online grief-related activities is the ease with which those bereaved can become preoccupied with the relationship, hindering the meaning-making process [21,38].

Positive and negative experiences with continuing bonds were often intertwined. Wood [23] found that negative thoughts about the deceased's personality or behaviour initially evoked distress but with reflection and perspective, understanding and personal growth could be achieved. This was echoed by Maple [16], who found that parents could initially be distressed at the ongoing 'presence' of their deceased child but in time came to enjoy these experiences. Nonetheless, participants reported a bitter 'aftertaste' associated with positive thoughts about the deceased as they tended to trigger difficult emotions and memories [23]. Remembering the more holistic picture (both good and bad) was beneficial in making sense of the suicide and relieving responsibility and guilt in those bereaved by suicide [23,39].

\subsection{Limitations}

The studies included in the review and the review itself entailed a few limitations. Most studies were qualitative, or cross-sectional (of relatively poor quality) with primarily female participants from western countries, making the impact of culture, sex and time since bereavement difficult to discern. This highlights the need for international research involving representative samples. Also, further studies involving control groups or adopting 
community-based longitudinal designs may enable researchers to capture the experiences of continuing bonds over time. While the review involved searches in six databases, future reviews can broaden the scope by including more databases as well as grey literature, which may increase, for example, the likelihood of finding negative experiences in the context of continuing bonds.

\subsection{Implications}

A better understanding of continuing bonds in suicide bereavement may inform future interventions and enable service providers to deliver more accurate and targeted support. In particular, the review highlights the substantial impact that societal expectations and stigma continue to have on the experience of grief in suicide bereaved individuals [40]. The findings of this review may direct future studies and help to characterise the experiences of continuing bonds in people bereaved by suicide with greater clarity.

\section{Conclusions}

People bereaved by suicide commonly experience continuing bonds and generally interpret these as positive experiences. Factors such as time since bereavement, social and cultural expectations, sex of the bereaved person, and the ability of the bereaved to make meaning of the death may influence how continuing bonds manifest and whether they are experienced as positive or negative (although further research is needed). Postvention efforts should consider the process of meaning-making in creating the basis for positive continuing bonds and should attempt to address the stigma and societal expectations surrounding suicide bereavement. Future studies should involve representative samples, compare with continuing bonds after other causes of death, and investigate continuing bonds in suicide bereavement longitudinally.

Author Contributions: Conceptualization, K.A., K.K. and R.G.; methodology, R.G., K.K. and K.A.; software, R.G.; validation, K.K. and K.A.; formal analysis, R.G. and K.A.; investigation, R.G.; data curation, R.G. and K.A.; writing —original draft preparation, R.G.; writing-review and editing, R.G., K.K. and K.A.; supervision, K.K. and K.A.; project administration, K.A. All authors have read and agreed to the published version of the manuscript.

Funding: K.A. was supported by a National Health and Medical Research Council Early Career Fellowship (GNT1157796). The funder had no role in the design of the study, in the collection, analyses, or interpretation of data, in the writing of the manuscript, or in the decision to publish the results.

Institutional Review Board Statement: Not applicable.

Conflicts of Interest: The authors declare no conflict of interest.

\section{Appendix A}

Table A1. Quality assessment ${ }^{1}$ of quantitative studies.

\begin{tabular}{lcc}
\hline \multicolumn{1}{c}{ Topic } & Jahn \& Spencer-Thomas, & 2014 [19] \\
\hline \multicolumn{1}{c}{ Selection } & \\
\hline (1) Representativeness of the exposed cohort & \\
\hline (a) Truly representative (one star) & $X$ \\
\hline (b) Somewhat representative (one star) & \\
\hline (c) Selected group & \\
\hline (d) No description & \\
\hline (2) Selection of the non-exposed cohort & $\mathrm{X} / \mathrm{a}$ \\
\hline (a) Drawn from the same community as the exposed cohort (one star) & $\mathrm{X}$ \\
\hline
\end{tabular}


Table A1. Cont.

Topic

Jahn \& Spencer-Thomas, 2014 [19]

Levi-Belz, 2017 [24]

(b) Drawn from a different source

(c) No description

(3) Ascertainment of exposure

(a) Secure record (e.g., surgical record) (one star)

(b) Structured interview (one star)

(c) Written self-report

$\mathrm{X}$

$\mathrm{X}$

(d) No description

(e) Other

(4) Demonstration that outcome of interest was not present at start of study
(a) Yes (one star)
$\mathrm{X}$
$\mathrm{X}$
(b) No

\section{Comparability}

(1) Comparability of cohorts on the basis of the design or analysis controlled for confounders
(a) The study controls for age, sex and marital status (one star)
$\mathrm{X}$
(b) Study controls for other factors (list) (one star)
$X$
(c) Controls are not comparable

\section{Outcome}

(1) Assessment of outcome

(a) Independent blind assessment (one star)

(b) Record linkage (one star)

(c) Self-report

$X$

$\mathrm{X}$

(d) No description

(e) Other

(2) Was follow-up long enough for outcomes to occur
(a) Yes (one star)
$\mathrm{X}$
(b) No
Indicate the mean duration of follow-up and a brief rationale for the assessment above

(3) Adequacy of follow-up of cohorts

(a) Complete follow-up, all subjects accounted for (one star)

(b) Subjects lost to follow-up unlikely to introduce bias, number lost less than or equal to $20 \%$ or description of those lost suggested no different from those followed (one star)

(c) Follow-up rate less than $80 \%$ and no description of those lost

\begin{tabular}{llll}
\hline (d) No statement & & $X$ & $X$ \\
& Stars & & \\
\hline Selection & & 1 & 2 \\
\hline Comparability & & 1 & 2 \\
\hline Outcome & Rating & Poor & Poor \\
\hline
\end{tabular}

\footnotetext{
${ }^{1}$ Newcastle-Ottawa Quality Assessment Form for Cohort Studies [12]. Note: A study can be given a maximum of one star for each numbered item within the Selection and Outcome categories. A maximum of two stars can be given for Comparability. Thresholds for converting the Newcastle-Ottawa scales to AHRQ standards (good, fair and poor): Good quality: 3 or 4 stars in selection domain AND 1 or 2 stars in comparability domain AND 2 or 3 stars in outcome/exposure domain. Fair quality: 2 stars in selection domain AND 1 or 2 stars in comparability domain AND 2 or 3 stars in outcome/exposure domain. Poor quality: 0 or 1 star in selection domain OR 0 stars in comparability domain OR 0 or 1 stars in outcome/exposure domain.
} 


\section{Appendix B}

Table A2. Quality assessment ${ }^{1}$ of qualitative studies.

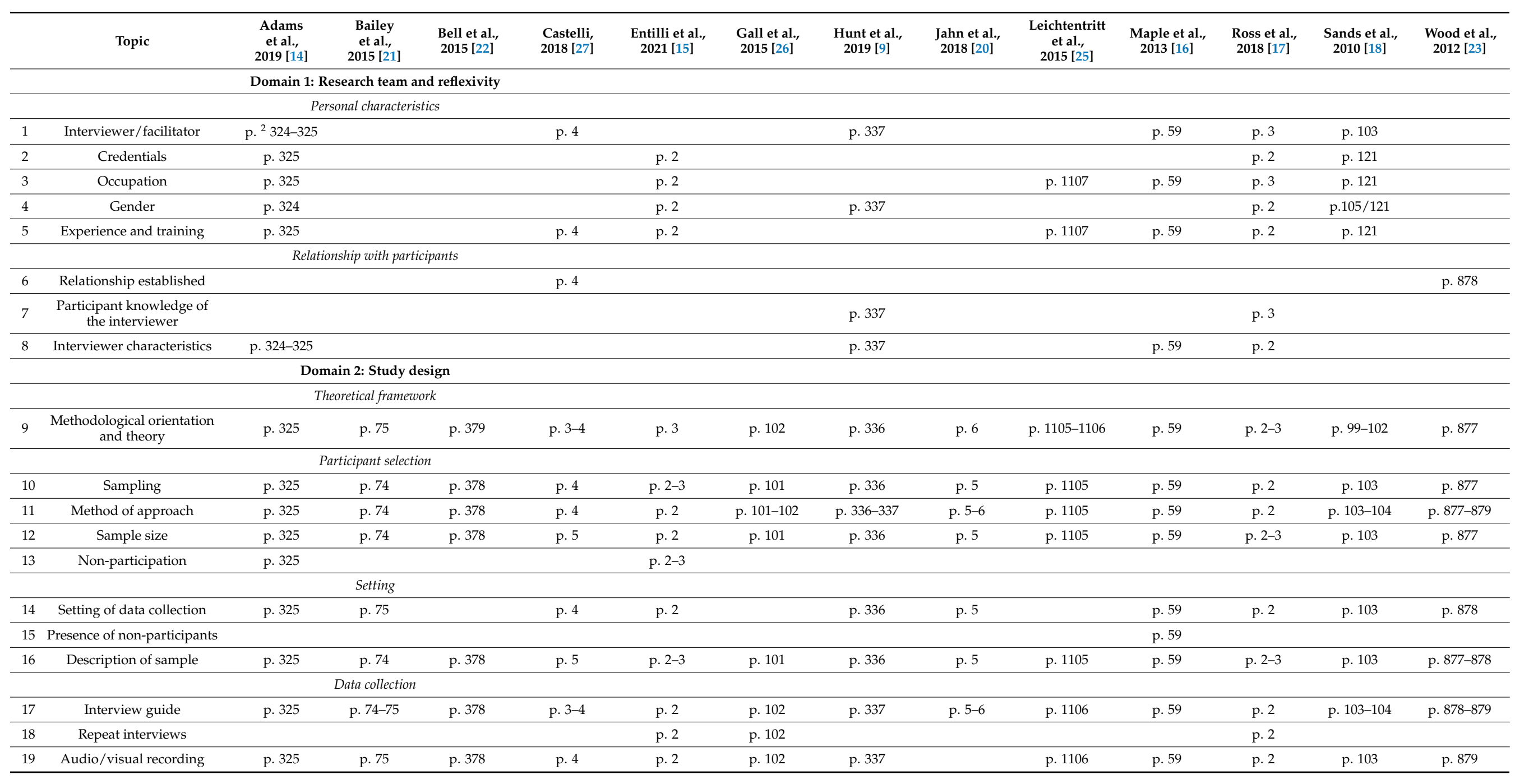


Table A2. Cont.

\begin{tabular}{|c|c|c|c|c|c|c|c|c|c|c|c|c|c|c|}
\hline & Topic & $\begin{array}{c}\text { Adams } \\
\text { et al., } \\
2019 \text { [14] }\end{array}$ & $\begin{array}{c}\text { Bailey } \\
\text { et al., } \\
2015 \text { [21] }\end{array}$ & $\begin{array}{l}\text { Bell et al., } \\
2015 \text { [22] }\end{array}$ & $\begin{array}{l}\text { Castelli, } \\
2018[27]\end{array}$ & $\begin{array}{l}\text { Entilli et al., } \\
2021 \text { [15] }\end{array}$ & $\begin{array}{l}\text { Gall et al., } \\
2015 \text { [26] }\end{array}$ & $\begin{array}{l}\text { Hunt et al., } \\
2019 \text { [9] }\end{array}$ & $\begin{array}{l}\text { Jahn et al., } \\
2018 \text { [20] }\end{array}$ & $\begin{array}{l}\text { Leichtentritt } \\
\text { et al., } \\
2015 \text { [25] }\end{array}$ & $\begin{array}{l}\text { Maple et al., } \\
2013 \text { [16] }\end{array}$ & $\begin{array}{l}\text { Ross et al., } \\
2018 \text { [17] }\end{array}$ & $\begin{array}{l}\text { Sands et al., } \\
2010[18]\end{array}$ & $\begin{array}{l}\text { Wood et al., } \\
2012 \text { [23] }\end{array}$ \\
\hline 20 & Field notes & & & & p. 4 & & & & & p. 1107 & & & & \\
\hline 21 & Duration & p. 325 & p. 75 & & p. 4 & p. 3 & p. 101 & & & p. 1106 & p. 59 & p. 2 & p. 103-104 & p. 878 \\
\hline 22 & Data saturation & & & & p. 5 & p. 2 & & p. 338 & & & & p. 2-3 & & \\
\hline \multirow[t]{3}{*}{23} & Transcripts returned & & & & p. 5 & & & & & & & & & \\
\hline & & Domain & Analysis a & findings & & & & & & & & & & \\
\hline & & & Data analysi & & & & & & & & & & & \\
\hline 25 & $\begin{array}{l}\text { Description of } \\
\text { the coding tree }\end{array}$ & p. 326 & & & & p. $3-6$ & & p. $337-338$ & & & & & & \\
\hline 26 & Derivation of themes & p. 325 & p. 75 & p. 378 & p. 4 & p. 3 & p. 102 & p. 337 & p. 6 & p. 1107 & p. 59 & p. 3 & p. 105-106 & p. 879 \\
\hline 27 & Software & & & & p. 4 & p. 3 & & & p. 6 & & & p. 3 & & \\
\hline \multirow[t]{2}{*}{28} & Participant checking & & & & p. 5 & & & p. 337 & & & & & & \\
\hline & & & Reporting & & & & & & & & & & & \\
\hline 29 & Quotations presented & p. $326-329$ & p. $76-81$ & p. $380-385$ & p. 6-14 & p. 3-6 & p. $102-108$ & p. 338-342 & p. 6-10 & p. $1108-1114$ & p. 60-64 & p. 3-6 & p. $107-113$ & p. $880-888$ \\
\hline 30 & Data and findings consistent & p. 329-331 & p. $81-83$ & p. 383-386 & p. 14-17 & p. 6-10 & p. $108-110$ & p. $342-343$ & p. 10-11 & p. $1115-1117$ & p. 65-67 & p. 7-8 & p. 114-117 & p. $888-894$ \\
\hline \multirow[t]{6}{*}{32} & Clarity of minor themes & & & & & p. 3-6 & & p. $338-342$ & & & & & p. 105-106 & p. $880-888$ \\
\hline & & & Rating & & & & & & & & & & & \\
\hline & $\begin{array}{l}\text { Domain 1: Research team } \\
\text { and reflexivity }\end{array}$ & $6 / 8(75 \%)$ & $0 / 8(0 \%)$ & $0 / 8(0 \%)$ & $\begin{array}{c}3 / 8 \\
(38 \%) \\
\end{array}$ & $\begin{array}{c}4 / 8 \\
(50 \%) \\
\end{array}$ & $\begin{array}{l}0 / 8 \\
(0 \%)\end{array}$ & $\begin{array}{l}4 / 8 \\
(50 \%) \\
\end{array}$ & $\begin{array}{l}0 / 8 \\
(0 \%)\end{array}$ & $\begin{array}{l}2 / 8 \\
(25 \%) \\
\end{array}$ & $\begin{array}{l}4 / 8 \\
(50 \%) \\
\end{array}$ & $\begin{array}{c}7 / 8 \\
(88 \%) \\
\end{array}$ & $\begin{array}{l}5 / 8 \\
(63 \%) \\
\end{array}$ & $\begin{array}{l}1 / 8 \\
(13 \%) \\
\end{array}$ \\
\hline & Domain 2: Study design & $10 / 15(67 \%)$ & $\begin{array}{l}9 / 15 \\
(60 \%)\end{array}$ & $7 / 15(47 \%)$ & $12 / 15(80 \%)$ & $12 / 15(80 \%)$ & $\begin{array}{l}9 / 15 \\
(60 \%) \\
\end{array}$ & $\begin{array}{l}9 / 15 \\
(60 \%) \\
\end{array}$ & $\begin{array}{l}7 / 15 \\
(47 \%) \\
\end{array}$ & $\begin{array}{l}9 / 15 \\
(60 \%) \\
\end{array}$ & $\begin{array}{l}10 / 15 \\
(67 \%) \\
\end{array}$ & $\begin{array}{l}11 / 15 \\
(73 \%)\end{array}$ & $\begin{array}{l}9 / 15 \\
(60 \%) \\
\end{array}$ & $\begin{array}{l}9 / 15 \\
(60 \%)\end{array}$ \\
\hline & $\begin{array}{l}\text { Domain 3: Analysis } \\
\text { and findings }\end{array}$ & $6 / 9(67 \%)$ & $\begin{array}{c}4 / 9 \\
(44 \%)\end{array}$ & $4 / 9(44 \%)$ & $\begin{array}{l}6 / 9 \\
(67 \%) \\
\end{array}$ & $8 / 9(89 \%)$ & $\begin{array}{l}5 / 9 \\
(56 \%) \\
\end{array}$ & $\begin{array}{c}8 / 9 \\
(89 \%) \\
\end{array}$ & $\begin{array}{l}6 / 9 \\
(67 \%)\end{array}$ & $\begin{array}{c}4 / 9 \\
(44 \%)\end{array}$ & $\begin{array}{c}4 / 9 \\
(44 \%) \\
\end{array}$ & $\begin{array}{l}6 / 9 \\
(67 \%) \\
\end{array}$ & $\begin{array}{l}6 / 9 \\
(67 \%) \\
\end{array}$ & $\begin{array}{l}6 / 9 \\
(67 \%) \\
\end{array}$ \\
\hline & Total & $22 / 32(69 \%)$ & $\begin{array}{l}13 / 32 \\
(41 \%)\end{array}$ & $11 / 32(34 \%)$ & $21 / 32(66 \%)$ & $24 / 32(75 \%)$ & $\begin{array}{l}14 / 32 \\
(44 \%)\end{array}$ & $\begin{array}{l}21 / 32 \\
(66 \%)\end{array}$ & $\begin{array}{l}13 / 32 \\
(41 \%)\end{array}$ & $\begin{array}{l}15 / 32 \\
(67 \%)\end{array}$ & $\begin{array}{l}18 / 32 \\
(56 \%)\end{array}$ & $\begin{array}{l}24 / 32 \\
(75 \%)\end{array}$ & $\begin{array}{l}20 / 32 \\
(63 \%)\end{array}$ & $\begin{array}{l}16 / 32 \\
(50 \%)\end{array}$ \\
\hline
\end{tabular}

${ }^{1}$ Consolidated Criteria for Reporting Qualitative Research (COREQ) [13]. ${ }^{2}$ In this table, "p." refers to page numbers. 


\section{References}

1. World Health Organisation. Suicide; [Updated 17 June 2021]; World Health Organisation: Geneva, Switzerland, 2021. Available online: https:/ / www.who.int/news-room/fact-sheets/detail/suicide (accessed on 6 September 2021).

2. Andriessen, K.; Rahman, B.; Draper, B.; Dudley, M.; Mitchell, P.B. Prevalence of exposure to suicide: A meta-analysis of population-based studies. J. Psychiatr. Res. 2017, 88, 113-120. [CrossRef] [PubMed]

3. Pitman, A.; Osborn, D.; King, M.; Erlangsen, A. Effects of suicide bereavement on mental health and suicide risk. Lancet Psychiatry 2014, 1, 86-94. [CrossRef]

4. Jordan, J.R.; McIntosh, J.L. Grief after Suicide: Understanding the Consequences and Caring for the Survivors; Routledge: New York, NY, USA, 2011.

5. Boerner, K.; Stroebe, M.; Schut, H.; Wortman, C.B. Theories of Grief and Bereavement. In Encyclopedia of Geropsychology; Pachana, N.A., Ed.; Springer: Singapore, 2015; pp. 1-10.

6. $\quad$ Klass, D.; Silverman, P.R.; Nickman, S.; Nickman, S.L. Continuing Bonds: New Understandings of Grief; Taylor \& Francis: Washington, DC, USA, 1996.

7. Klass, D.; Steffen, E. Continuing Bonds in Bereavement: New Directions for Research and Practice; Routledge: New York, NY, USA, 2017.

8. Neimeyer, R.A.; Baldwin, S.A.; Gillies, J. Continuing bonds and reconstructing meaning: Mitigating complications in bereavement. Death Stud. 2006, 30, 715-738. [CrossRef]

9. Hunt, Q.A.; Young, T.A.; Hertlein, K.M. The process of long-term suicide bereavement: Responsibility, familial support, and meaning making. Contemp. Fam. Ther. 2019, 41, 335-346. [CrossRef]

10. Rubin, S.S.; Nadav, O.B.; Malkinson, R.; Koren, D.; Goffer-Shnarch, M.; Michaeli, E. The two-track model of bereavement questionnaire (TTBQ): Development and validation of a relational measure. Death Stud. 2009, 33, 305-333. [CrossRef] [PubMed]

11. Page, M.J.; McKenzie, J.E.; Bossuyt, P.M.; Boutron, I.; Hoffmann, T.C.; Mulrow, C.D.; Shamseer, L.; Tetzlaff, J.M.; Akl, E.A.; Brennan, S.E.; et al. The PRISMA 2020 statement: An updated guideline for reporting systematic reviews. BMJ 2021, 372, n71. [CrossRef]

12. Wells, G.; Shea, B.; O'Connell, D.; Peterson, J.; Welch, V.; Losos, M.; Tugwell, P. Newcastle-Ottawa Quality Assessment Scale Cohort Studies. University of Ottawa: Ottawa, ON, Canada, n.d. Available online: https://www.ncbi.nlm.nih.gov/books/NBK9 9082/bin/appb-fm4.pdf (accessed on 6 September 2021).

13. Tong, A.; Sainsbury, P.; Craig, J. Consolidated criteria for reporting qualitative research (COREQ): A 32-item checklist for interviews and focus groups. Int. J. Qual. Health Care 2007, 19, 349-357. [CrossRef]

14. Adams, E.; Hawgood, J.; Bundock, A.; Kõlves, K. A phenomenological study of siblings bereaved by suicide: A shared experience. Death Stud. 2019, 43, 324-332. [CrossRef]

15. Entilli, L.; Ross, V.; De Leo, D.; Cipolletta, S.; Kõlves, K. Experiences of parental suicide-bereavement: A longitudinal qualitative analysis over two years. Int. J. Environ. Res. Public Health 2021, 18, 564. [CrossRef]

16. Maple, M.; Edwards, H.E.; Minichiello, V.; Plummer, D. Still part of the family: The importance of physical, emotional and spiritual memorial places and spaces for parents bereaved through the suicide death of their son or daughter. Mortality 2013, 18, 54-71. [CrossRef]

17. Ross, V.; Kõlves, K.; Kunde, L.; de Leo, D. Parents' experiences of suicide-bereavement: A qualitative study at 6 and 12 months after loss. Int. J. Environ. Res. Public Health 2018, 15, 618. [CrossRef] [PubMed]

18. Sands, D.; Tennant, M. Transformative learning in the context of suicide bereavement. Adult Educ. Q. 2010, 60, 99-121. [CrossRef]

19. Jahn, D.; Spencer-Thomas, S. Continuing bonds through after-death spiritual experiences in individuals bereaved by suicide. J. Spiritual. Ment. Health 2014, 16, 311-324. [CrossRef]

20. Jahn, D.; Spencer-Thomas, S. A qualitative examination of continuing bonds through spiritual experiences in individuals bereaved by suicide. Religions 2018, 9, 248. [CrossRef]

21. Bailey, L.; Bell, J.; Kennedy, D. Continuing social presence of the dead: Exploring suicide bereavement through online memorialisation. New Rev. Hypermedia Multimed. 2015, 21, 72-86. [CrossRef]

22. Bell, J.; Bailey, L.; Kennedy, D. 'We do it to keep him alive': Bereaved individuals' experiences of online suicide memorials and continuing bonds. Mortality 2015, 20, 375-389. [CrossRef]

23. Wood, L.; Byram, V.; Gosling, A.; Stokes, J. Continuing bonds after suicide bereavement in childhood. Death Stud. 2012, 36, 873-898. [CrossRef] [PubMed]

24. Levi-Belz, Y. Relationship with the deceased as facilitator of posttraumatic growth among suicide-loss survivors. Death Stud. 2017, 41, 376-384. [CrossRef]

25. Leichtentritt, R.D.; Yerushalmi, A.; Barak, A. Characteristics of the ongoing bond. Br. J. Soc. Work 2015, 45, 1102-1118. [CrossRef]

26. Gall, T.L.; Henneberry, J.; Eyre, M. Spiritual beliefs and meaning-making within the context of suicide bereavement. J. Study Spiritual. 2015, 5, 98-112. [CrossRef]

27. Castelli Dransart, D.A. Spiritual and religious issues in the aftermath of suicide. Religions 2018, 9, 153. [CrossRef]

28. Krysinska, K.; Andriessen, K.; Corveleyn, J. Religion and spirituality in online suicide bereavement: An analysis of online memorials. Crisis 2014, 35, 349-356. [CrossRef] [PubMed]

29. Čepulienè, A.A.; Pučinskaitè, B.; Spangelytè, K.; Skruibis, P.; Gailienè, D. Spirituality and religiosity during suicide bereavement: A qualitative systematic review. Religions 2021, 12, 766. [CrossRef]

30. Parker, C.M.; Laungani, P.; Young, B. Death and Bereavement across Cultures; Routledge: New York, NY, USA, 1997. 
31. Stroebe, M.; Schut, H. The dual process model of coping with bereavement: A decade on. Omega 2010, 61, 273-289. [CrossRef] [PubMed]

32. Foster, T.L.; Gilmer, M.J.; Davies, B.; Dietrich, M.S.; Barrera, M.; Fairclough, D.L.; Vannatta, K.; Gerhardt, C.A. Comparison of continuing bonds reported by parents and siblings after a child's death from cancer. Death Stud. 2011, 35, 420-440. [CrossRef] [PubMed]

33. Grad, O.T.; Treven, M.; Krysinska, K. Suicide Bereavement and Gender. In Postvention in Action: The International Handbook of Suicide Bereavement; Andriessen, K., Krysinska, K., Grad, O., Eds.; Hogrefe: Gottingen, Germany, 2017; pp. $39-49$.

34. Milman, E.; Neimeyer, R.A.; Fitzpatrick, M.; MacKinnon, C.J.; Muis, K.R.; Cohen, S.R. Rumination moderates the role of meaning in the development of prolonged grief symptomatology. J. Clin. Psychol. 2019, 75, 1047-1065. [CrossRef]

35. Root, B.L.; Exline, J.J. The role of continuing bonds in coping with grief: Overview and future directions. Death Stud. 2014, 38, 1-8. [CrossRef]

36. Stroebe, M.; Schut, H.; Boerner, K. Continuing bonds in adaptation to bereavement: Toward theoretical integration. Clin. Psychol. Rev. 2010, 30, 259-268. [CrossRef]

37. Krysinska, K.; Andriessen, K. Suicide Bereavement Online: Sharing Memories, Seeking Support, and Exchanging Hope. In Suicide Prevention and New Technologies. Evidence Based Practice; Mishara, B., Kerkhof, A., Eds.; Palgrave-Macmillan: New York, NY, USA, 2014; pp. 150-165.

38. Krysinska, K.; Andriessen, K. Online memorialization and grief after suicide: An analysis of suicide memorials on the Internet. Omega 2015, 71, 19-47. [CrossRef]

39. Sands, D. A tripartite model of suicide grief: Meaning-making and the relationship with the deceased. Grief Matters 2009, 12, 10-17.

40. Evans, A.; Abrahamson, K. The influence of stigma on suicide bereavement: A systematic review. J. Psychosoc. Nurs. Ment. Health Serv. 2020, 58, 21-27. [CrossRef] [PubMed] 